\title{
PFO and cryptogenic stroke: Do we finally have closure?
}

\section{Introduction}

In up to 40 percent of patients with acute ischemic stroke, the etiology remains uncertain [1]. The association of a PFO with stroke in a young woman with cerebral arterial embolism was first described in 1877 [2]. Lechat et al. later described the high prevalence of PFO in adults younger than 55 years of age when no identifiable cause of stroke could be identified [3]. They suggested that paradoxical embolism through the PFO may be responsible for stroke more often than had been suspected. Further studies suggested that the size and the degree of shunting as identified by transesophageal echocardiography were important determinates of ischemic stroke and recurrent strokes. Furthermore, the presence of both a patent foramen ovale and atrial septal aneurysm were determinants of a substantial risk for recurrent stroke in individuals with a previous stroke of unknown origin despite the use of aspirin [4]. When aspirin was compared to warfarin in patients with PFO, there was no difference in the rates of adverse events or in the time to the first event, thus raising the speculation that closure of the PFO may decrease the incidence of recurrent events over medical therapy alone [5].

In 1998, the Amplatzer PFO Occluder received European $\mathrm{CE}$ mark for patients with a history of stroke and PFO diagnosed by echocardiography with right to left shunting during Valsalva maneuver. It has since been made available in more than 60 countries. The European approval was based on observational studies and case series rather than randomized trial data. For years the management strategy for patients with a clinically significant PFO has been a subject of intense controversy due to limited randomized trial data, which have been debatable and inconsistent. To date there have been three randomized trials of closure versus medical therapy in this patient population all of which were initiated after the CE mark approval.
The first randomized trial (CLOSURE I) David L Fischman?, Rodney Bell ${ }^{2}$, Nicholas was published in 2012. In a study of 909 Ruggiero and Michael P Savage ${ }^{1 *}$ patients randomized 1:1 to transcatheter ${ }^{1}$ Department of Medicine, Sidney Kimmel Medical College, PFO closure using the StarFlex Septal Thomas Jefferson University, USA Closure System (NMT Medical Inc., Thomas Jefferson University, USA

Boston, MA) with 6 months of dual- Michael.Savage@jefferson.edu antiplatelet therapy composed of aspirin Submitted: 6 February 2017 and clopidogrel, or to best medical therapy Accepted: 8 February 2017 (aspirin, warfarin, or a combination of the two) there was no significant differences in the primary endpoint of recurrent stroke (3.2\% for transcatheter closure vs 3.5\% for medical therapy; $\mathrm{P}=0.80)$ or TIA $(3.2 \%$ vs $4.6 \% ; \quad \mathrm{P}=0.31)$ at 2-year follow-up (composite endpoint of $5.8 \%$ vs $7.7 \%$; $\mathrm{P}=0.28)$. The incidence of both major vascular complications $(3.2 \%$ vs $0 \%$; $\mathrm{P}<0.001)$ and atrial fibrillation $(5.7 \%$ vs $0.7 \%$; $<<0.001)$ were significantly higher in the transcatheter PFO closure group [6].

The PC trial was the second of the three trials [7]. This was a physician-initiated prospective, multicenter randomized controlled trial of the Amplatzer PFO Occluder conducted outside of the United States. Between 2000 and 2009, a total of 414 patients were enrolled. This trial did not demonstrate a statistically significant reduction in the primary composite endpoint of death, nonfatal stroke, TIA or peripheral embolism.

In August 2003, the RESPECT trial enrolled its first patient. The largest randomized PFO trial was designed to demonstrate superiority of the Amplatzer PFO occluder over medical therapy in reducing a recurrent ischemic stroke. Enrollment was completed in 2011 when the 25th pre-specified primary endpoint was observed among a total of 980 patients enrolled. Over a median duration of followup of 2.1 years, the primary endpoint (all recurrent strokes) by intention-to-treat analysis was not significantly different between the PFO closure and medical therapy arms. 9 Patients in the closure group and 16 in the medical-therapy group had a recurrence of stroke (HR 0.49, 95\% 
CI $0.22-1.11, \mathrm{p}=0.08)$. On per-protocol analysis, there was a benefit favoring PFO closure with 6 events in the closure group and 14 events in the medical therapy arm (HR 0.37; 95\% CI 0.14-0.96, p=0.03).

Subgroup analysis revealed a trend towards a benefit in patients with large shunts and atrial septal aneurysms. Atrial fibrillation was noted in $0.6 \%$ of patients in both arms and other procedural complications were infrequent [8].

Furthermore, there was no difference noted in all-cause strokes between PFO and medical management arms on extended follow-up (mean -5 years) $(\mathrm{p}=0.16)$. A blinded assessment was performed of the recurrent strokes, and one third of them were noted to be noncryptogenic due to mechanisms such as smallvessel atherosclerotic disease and atrial fibrillation. In those with a recurrent cryptogenic stroke, a 54\% relative risk reduction in favor of $\mathrm{PFO}$ closure was noted (HR 0.46, $\mathrm{p}=0.042$ ). On further long-term follow-up (mean 5.9 years), there was a $45 \%$ risk reduction in recurrent ischemic strokes (18 vs 28) in favor of PFO closure (HR 0.55, 95\% CI 0.31-1.0, $\mathrm{p}=0.046)$ [9].

In May 2016, the FDA advisory panel voted in favour of approving the Amplatzer device, based on the extended follow-up data, albeit by a narrow margin due to the less than conclusive nature of the RESPECT trial. With respect to efficacy, the vote was 9-7 in favor. Nearly 14 years after the initiation of the RESPECT trial, the FDA followed through with the panel's recommendation and approved on October 28, 2016 the Amplatzer PFO occluder for patients between 18 and 60 years old with a cryptogenic stroke. The FDA emphasized the need for a strong collaboration between neurologists and cardiologists to exclude other causes of strokes. Was the FDA premature in its decision or long overdue?

During the 8 years of trial enrollment the clinical protocol underwent 5 revisions, many of which were intended to address the slow enrollment. Despite these revisions, the RESPECT trial failed to find a benefit for closure according to its original primary endpoint. It was only after additional years of follow-up did a difference emerge in favor of PFO closure over best medical therapy. As part of the medical therapy arm, subjects could be treated with one of a number of antithrombotic therapies that included aspirin alone, warfarin alone, clopidogrel alone, aspirin plus dipyridamole, or aspirin plus clopidogrel. Thus, the study never truly defined or tested the best medical therapy. In addition, greater than $90 \%$ of subjects in the device group were taking antithrombotic therapies (predominantly antiplatelet agents) throughout the study [9]. The rate of subject discontinuation was relatively high for the entire enrolled population and was higher in the medical arm (30.1\% vs $18.2 \%)$ testing the robustness of this trial in which there were relatively few primary endpoints. Previous studies had suggested that individuals with large PFO and/or an atrial septal defect were at increased risk for a recurrent stroke. Although there was a trend towards a benefit in these patient subgroups, the small overall event rates prohibited specification of device use in these patients.

In August, prior to the FDA approval, the American Academy of Neurology (AAN) recognizing the limitations of the RESPECT trial published a practice advisory stating that it does not recommend "routine" PFO closure for stroke prevention. The advisory urged physicians to educate patients on the high prevalence of PFO's and how rarely they have been proven to cause recurrent stroke [10]. Now that there is an FDA approved device, we are confronted with the challenge of determining which patients might benefit from PFO closure. How do we achieve the appropriate clinical equipoise between benefit and risk, between device overutilization and underutilization?

Therapeutic uncertainty is inherent in decisions in patients with PFO and cryptogenic stroke. Since both PFO's and strokes are common, the clinical challenge is to determine which strokes are likely related to the PFO. In the interest of best serving our patients, some common sense suggestions seem evident. First, stick to the script. PFO closure is indicated for patients less than 60 years of age who have suffered an ischemic stroke with no other apparent proximate cause. The device is not approved for patients with TIA symptoms in the absence of stroke. Older patients are more likely to have alternative etiologies for their stroke and the discovery of a PFO during their workup is more likely to be a coincidental, unrelated finding. Second, the decision to perform PFO closure should not be left to the interventional cardiologist alone given the propensity for some to behave like children with a new hammer where everything looks like a nail. It is imperative to heed the FDA recommendations for collaboration between neurologists and cardiologists in the evaluation and treatment of these patients. Taking precedence from the "heart team" approach for TAVR involving cardiologists and cardiothoracic surgeons, perhaps a "heart-brain team" approach should be mandated.

Given all the noted limitations of the trial data despite nearly two decades of investigation, it's uncertain that we have truly reached "closure" on PFO device implantation in the setting of cryptogenic stroke. The key to optimize outcomes is to establish a coordinated team of neurologists and cardiologists to ensure the best decision is made for each and every individual patient.

\section{References}

1. Sacco RL, Ellenberg JH, Mohr JP, et al. Infarcts of undetermined cause: the NINCDS Stroke DataBank. Ann. Neurol. 25, 382-390 (1989). 
2. Cohnheim J. Thrombose und embolie. Vorlesungren Uber Allgemenie Pathologie.

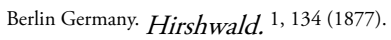

3. Lechat JL, Mas JL, Lascault G, et al. Prevalence of patent foramen ovale in patients with stroke. N. Engl. J. Med. 318, 1148-1152 (1988).

4. Mas JL, Arquizan C, Lamy C, et al. Patent Foramen Ovale and Atrial Septal Aneurysm Study Group. Recurrent cerebrovascular events associated with patent foramen ovale, atrial septal aneurysm, or both. N. Engl. J. Med. 345, 1740-1746 (2001).

5. Homma S, Sacco RL, Di Tullio MR, Sciacca RR, Mohr JP. PFO in Cryptogenic Stroke Study (PICSS) Investigators. Effect of medical treatment in stroke patients with patent foramen ovale: patent foramen ovale in cryptogenic stroke study. Circulation. 105, 2625-2631 (2002).

6. Furlan AJ, Reisman M, Massaro J, et al. CLOSURE I Investigators. Closure or Medical Theraoy for Cryptogenic Stroke with Patent Foramen Ovale. N. Engl. J. Med. 366, 991-999 (2012).

7. Meier B, Kalesan B, Mattle HP, et al, PC Trial Investigators. Percutaneous closure of patent foramen ovale in cryptogenic embolism. N. Engl. J. Med. ${ }^{368,}$ 1083-1091 (2013)

8. Carroll JD, Saver JL, Thaler DE, et al. RESPECT Investigators. Closure of patent foramen ovale versus medical therapy after cryptogenic stroke. N. Engl. J.

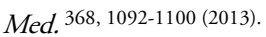

9. Amplatzer. PFO Occluder Executive Summary. Circulatory System Devices Panel ${ }^{(2016)}$.

10. Messe SR, Gronseth G, Kent DM, et al Practice advisory: Recurrent stroke with patent foramen ovale (update of practice parameter): Report of the guideline development, dissemination, and implementation subcommittee of the American Academy of Neurology. Neurology. 87, 815-821 (2016). 